\title{
Point Inoculation Method for Measuring Adult Plant Response of Wheat to Stripe Rust Infection
}

\author{
Willem H. P. Boshoff, ${ }^{1, \dagger}$ Renée Prins, ${ }^{1,2,3}$ Corneli de Klerk, ${ }^{2}$ Simon G. Krattinger, ${ }^{4}$ Cornelia M. Bender, ${ }^{1}$ Gerrie J. Maree, ${ }^{1}$ \\ Lisa Rothmann, ${ }^{1}$ and Zacharias A. Pretorius ${ }^{1}$ \\ ${ }^{1}$ Department of Plant Sciences, University of the Free State, Bloemfontein 9300, South Africa; ${ }^{2}$ CenGen (Pty.) Ltd., Worcester 6850 , \\ South Africa; ${ }^{3}$ Department of Genetics, Stellenbosch University, 7602, Stellenbosch, South Africa; and ${ }^{4}$ Biological and Environmental \\ Science and Engineering Division, King Abdullah University of Science and Technology, Thuwal, Saudi Arabia
}

\begin{abstract}
Depending on the pathogenicity of the stripe rust fungus Puccinia striiformis $\mathrm{f}$. sp. tritici, the nature of resistance in the wheat host plant, and the environment, a broad range of disease phenotypes can be expressed. Therefore, the phenotyping of partial adult plant stripe rust resistance requires reliable and repeatable procedures, especially under controlled conditions. In this study, the development of a flag leaf point inoculation method, which resulted in a $100 \%$ initial infection rate, is reported. Flag leaf inoculations were achieved by placing $6-\mathrm{mm}$ antibiotic test

paper discs, dipped into a urediniospore and water suspension and covered with water-proof plastic tape, on the adaxial side of leaves. Results from independent trials allowed for the statistical comparison of stripe rust lesion expansion rate in wheat entries that differ in resistance. The technique is inexpensive, reliable, and applicable to routine screening for adult plant response type, quantitative comparison of stripe rust progress, environmental influences, and pathogenicity of different isolates.
\end{abstract}

The short-lived effectiveness of many major genes for all stage resistance to rusts in wheat (Triticum aestivum $\mathrm{L}$.) has focused attention on more durable resistance types. When several minor genes or quantitative trait loci (QTLs) for adult plant resistance (APR) to the wheat rusts are combined, reduced disease levels occur, protection against a broader range of races follows, and expectations of durability increase (Dong et al. 2017; Klarquist et al. 2016; Mundt 2018; Pretorius et al. 2017; Singh et al. 2015). When deployed singly, many APR genes do not confer adequate resistance under high disease pressure; however, the combination of four or five minor genes can approach immunity or a high level of resistance (Singh et al. 2014). Chen (2005) mentioned that high-temperature APR to wheat stripe rust caused by Puccinia striiformis Westend. f. sp. tritici Erikss. is race-nonspecific and durable. Despite the appeal of APR, Michelmore et al. (2013) and Ellis et al. (2014) warned that our knowledge of this resistance type is incomplete and that certain components may indeed be race-specific and nondurable. The emphasis on APR to rust diseases, whether from a mechanistic or breeding perspective, requires accurate and reliable phenotyping. Although successful rust assessment is carried out in field nurseries across the world, certain germplasm collections may require more detailed and quantitative assessments of infection type and disease development over time. Reliable inoculation protocols in controlled conditions are also of great importance to enable and speed up the cloning of APR genes.

Several studies have investigated inoculation procedures for cereal rust assessment under controlled conditions. Andres and Wilcoxson (1984) developed a device for precise deposition of urediniospores on individual leaves of cereal plants, a system successfully used for quantifying components of resistance in wheat to the leaf rust pathogen $P$. triticina Erikss. (Kloppers and Pretorius 1997). Singh et al. (1996) described a convenient adhesive

${ }^{\dagger}$ Corresponding author: W. H. P. Boshoff; BoshoffWHP@ufs.ac.za

Funding: The South African Winter Cereal Trust and the National Research Foundation and University of Free State (UFS) (UID\#85943) are thanked for financial support.

Accepted for publication 27 November 2018.

C 2019 The American Phytopathological Society tape method for evaluating leaf rust response in flag leaves. In their approach, urediniospores were applied to sections of clear adhesive tape, which were then fixed to the upper surface of flag leaves. A further advantage of this technique was that more than one pathotype could be applied to the same leaf. Settling towers have also been successfully used for quantitative deposition of urediniospores on wheat seedlings or adult plants (Eyal et al. 1968; Mortensen et al. 1979). A challenge in using a settling tower is the uniform application of spores on plant parts such as erect stems or twisting flag leaves that are not fully or similarly fully exposed in a horizontal position.

Spray inoculations are also commonly used. Browder (1971) described an efficient inoculation system in which a suspension of urediniospores in light mineral oil, contained in gelatin capsules, is atomized onto foliage by custom-made inoculators. Hickey et al. (2012) and Riaz et al. (2016) applied spore-oil suspensions of, respectively, $P$. striiformis and $P$. triticina, by means of an air brush. Following procedures described by Pretorius et al. (2000, 2007) for flag leaf infection by $P$. striiformis and $P$. triticina, Bender et al. (2016) reported that a suspension of urediniospores of the stem rust pathogen $P$. graminis Pers. f. sp. tritici Erikss. \& Henning in water was most suitable for inoculating adult wheat plants in greenhouse experiments.

Broers and Lopez-Atilano (1994) placed agar blocks preinoculated with $P$. striiformis urediniospores on flag and flag-1 leaves of the highly susceptible cultivar Morocco to achieve uniform infections in quantitative studies of resistance components. However, the success rate of infections depended on the number of spores applied to the agar blocks. Milus et al. (2009) attained 72\% successful infections by inoculating wheat flag leaves with $P$. striiformis urediniospores suspended in an agar solution. More recently, Sørensen et al. (2016) compared different point inoculation methods for $P$. striiformis. In their experiments, treatments with urediniospores suspended in the engineered fluid Novec 7100, or mixed with Lycopodium spores in a dry inoculum product, gave a $100 \%$ infection rate. Although consistent infection was achieved for quantitative measurements, this method was developed for seedlings and required the horizontal fixing of leaves on a base plate.

Taking into account the advantages and disadvantages of the different methods, the objective of our study was to develop an uncomplicated yet reliable point inoculation method for assessing stripe rust response in wheat flag leaves. Primary requirements were a robust, 
easy to use technique, consistent infection, and assessment of infection type and/or rate of disease development.

\section{Materials and Methods}

Plant materials and growing conditions. The development of a stripe rust point inoculation method was investigated in two independent greenhouse trials. In the first trial the cultivars Avocet ' $S$ ' (susceptible control), Kariega (resistant control [South African spring wheat]) (Smit et al. 2010), and two experimental lines, MP152 + QYr.sgi-4A.(1) and Avocet $\mathrm{S}+$ QYr.sgi-4A.1 (hereafter referred to as MP152_4A and AvS_4A, respectively) were included. Both MP152_4A and AvS_4A were derived from a cross between Kariega and Avocet ' $S$ ' and express moderate levels of stripe rust resistance (slow rusting) under field conditions. Plants of the respective entries were grown in steam-sterilized soil in 2-liter pots, three plants per pot, set to an 18 to $22^{\circ} \mathrm{C}$ night/day temperature schedule in a greenhouse. Prevailing day length (approximately $12 \mathrm{~h}$ ) applied, with no additional lighting used in the greenhouse cubicles. After emergence, plants were watered twice a day with reverse-osmosis water and fertilized twice per week with Multifeed-Classic water-soluble fertilizer (Effekto NPK 19:8:16 [43]), at $2.5 \mathrm{mg} / \mathrm{liter}$ of water and $200 \mathrm{ml} /$ pot, for the duration of the trial.

In a second trial, the technique was validated using seven wheat entries known to vary in their field response to $P$. striiformis race $6 \mathrm{E} 22 \mathrm{~A}+$. These include the stripe rust susceptible entries Avocet 'S', Avocet 'R', Jupateco 'S', Krokodil, and JIC871, the moderately resistant entry Jupateco 'R', and the cultivar Trident ( $L r 37 / Y r 17$ ) Sr38), which expresses stripe rust APR under field conditions. Avocet ' $S$ ' and Avocet ' $R$ ', sources obtained from the Plant Breeding Institute, University of Sydney, Australia, are near-isogenic selections for the absence or presence of $\operatorname{YrA}$ from the Australian wheat cultivar Avocet (Wellings et al. 1988). Jupateco 'S' and Jupateco 'R', sources obtained from CIMMYT, were reselected for the absence or presence of the Lr34/Yr18/Sr57 gene, respectively (Singh 1992). Krokodil is a South African spring wheat cultivar (Smit et al. 2010), and JIC871, coded as entry W6241 in Prins et al. (2016), is a highly susceptible line from Ethiopian origin obtained from the Genome Resource Unit, Norwich Research Park, United Kingdom.

Inoculation experiments. In both trials, plants were inoculated with $P$. striiformis race $6 \mathrm{E} 22 \mathrm{~A}+$ when the majority were at midheading to mid-flowering (Zadoks growth stages 55 to 65) (Zadoks 1961). P. striiformis race 6E22A+ is commonly detected in South Africa after its first detection during 2005 and is virulent for $Y r 2$, Yr6, Yr7, Yr8, Yr11, Yr14, Yr17, Yr19, Yr25, and YrA (Visser et al. 2016). Inoculum consisted of $100 \mathrm{mg}$ of freshly collected urediniospores in $100 \mathrm{ml}$ of reverse-osmosis water without any added surfactant. Using a pair of forceps, a 6-mm antibiotic test paper disc was dipped into the spore layer on top of the suspension and placed in the middle of the adaxial surface, equidistant to the base and tip, of each flag leaf. Each disc was immediately covered with a strip of white-colored polyvinyl chloride electrical insulation tape (Nitto no. $21,0.2$ by $18 \mathrm{~mm}$, manufactured by Nitti Denko Corporation) placed across the width of the leaf. To facilitate easy removal, the adhesive tape was cut long enough to allow for a 1-cm extension on either side of the leaf. When covering discs, special care was taken to not put undue pressure onto the disc area itself in an effort to retain maximum moisture and contain the point of inoculation. Plants were kept at $10^{\circ} \mathrm{C}$ in the dark in a cold room for $24 \mathrm{~h}$ with a prevailing humidity of $87 \%$ (measured at 30-min intervals with an Onset data logger, model HOBO Pro v2) before placement in a greenhouse at 14 to $18^{\circ} \mathrm{C} \mathrm{night/day} \mathrm{set} \mathrm{temperatures.} \mathrm{The} \mathrm{tape} \mathrm{and} \mathrm{discs} \mathrm{were} \mathrm{carefully}$ removed $24 \mathrm{~h}$ later ( $48 \mathrm{~h}$ after inoculation).

In the first trial, four pots per entry, with five inoculated flag leaves per pot, were used in each trial replicate. On average, eight to 10 flag leaves were available for inoculation per pot, with only flag leaves between Zadoks 55 and 65 being considered. No trimming of noninoculated leaves or tillers was done. To evaluate the effect of post inoculation temperature on the expression of resistance, two treatments were applied. Two pots of each entry were kept in separate greenhouse cubicles, one with a lower mean temperature $\left(14.4^{\circ} \mathrm{C}\right.$ night and $17.8^{\circ} \mathrm{C}$ day) and the other with a higher mean temperature $\left(19.1^{\circ} \mathrm{C}\right.$ night and $23.7^{\circ} \mathrm{C}$ day). Temperature was measured at 30 min intervals with an Onset data logger (model HOBO 4-channel analog logger). No additional lighting was applied in greenhouse cubicles, and the prevailing day length was approximately $12 \mathrm{~h}$. Using an Apogee MQ-500 quantum meter, an average photosynthetic photon flux density of $1,350 \mu \mathrm{mol} /\left(\mathrm{m}^{2} \cdot \mathrm{s}\right)$ was measured in the greenhouse cubicles at midday. Pots were randomized on the respective greenhouse benches, and the trial was replicated in an independent experiment applying the same methodology.

In the second trial, which included two trial replicates, each replicate was represented by two pots per entry, with four inoculated flag leaves per pot maintained in greenhouse cubicles with mean night/ day temperature range of 14.6 and $18.5^{\circ} \mathrm{C}$, respectively.

Stripe rust assessment. In the first trial, the maximum length of visible lesion progression was measured at 3-day intervals from 7 to 22 days post inoculation (dpi) using a Mitutoyo digital caliper. A lesion was measured as the length between the visible chlorotic or necrotic margins, including the sporulating area if present, at opposite ends of an infection site. In the second trial the initial lesion size was measured at 7 and 12 dpi and thereafter every 5 days until the final measurements at $27 \mathrm{dpi}$.

Data analysis. Data were analyzed using NCSS: Statistical System (Hintze 2007). Data collected from the four pots per treatment in the first trial, representing two trial replicates with 10 data points per trial replicate, were pooled because the experiments did not differ significantly $(P \leq 0.05)$. Data were analyzed for normality and variance homogeneity using Shapiro-Wilk and Bartlett's tests, respectively. Nonlinear regression was used to determine the relationship between variables. The power model $\left(Y=A x^{b}\right)$ was used to describe the relationship between time (days after inoculation) and lesion length (mm). Lesion length data obtained 22 dpi were analyzed for variance (ANOVA; $y_{i j k}=\mu_{i}+\beta_{j}+\gamma_{i j}+\varepsilon_{i j k}$ ) including all variables. Mean separation was conducted using Fisher's unprotected test to determine the least significant difference at the 5\% significance level. Similarly, in the second trial, data obtained from the four pots per entry, representing two trial replicates with eight data points for each entry per trial replicate, were pooled because experiments did not differ significantly $(P \leq 0.05)$. Nonlinear regression analysis as described for trial 1 was applied using the logistic model $(Y=A /\{1+$ $B[\exp (-C x)]\}$ ) to describe the relationship between time (days after inoculation) and lesion length $(\mathrm{mm})$.

\section{Results}

The point inoculation technique developed for studying stripe rust infection on wheat flag leaves was highly reliable with a $100 \%$ infection rate achieved over trials, entries, and treatments. The appearance

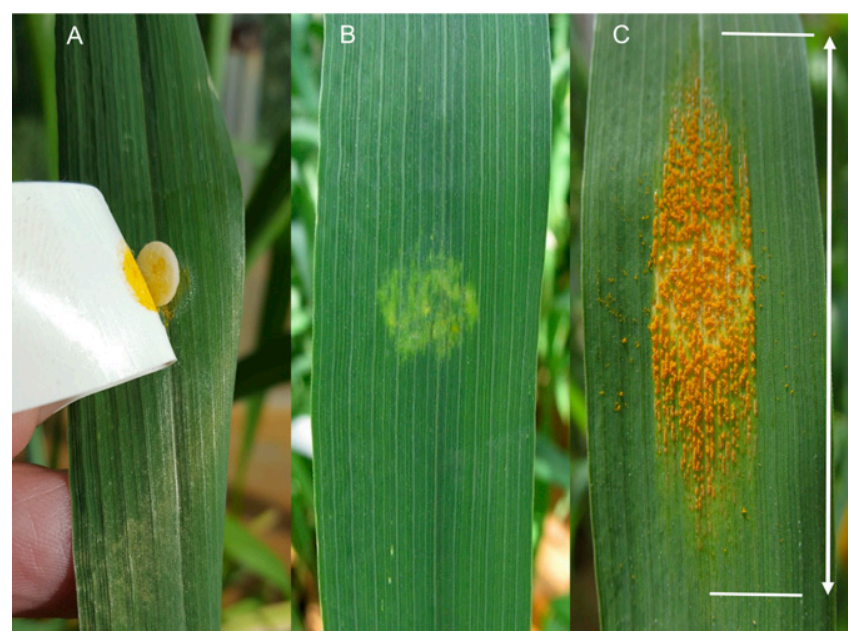

Fig. 1. A, Wheat flag leaf during removal of the plastic insulation tape and disc $48 \mathrm{~h}$ after inoculation with urediniospores of Puccinia striiformis f. sp. tritici race 6E22A+. B, Signs of $P$. striiformis initial infection visible as chlorosis 7 days post point inoculation. C, Expansion of stripe rust lesion length 15 days post inoculation. 
of the first symptoms of infection was visible as chlorosis 7 days after point inoculation on a flag leaf of the stripe rust susceptible cultivar Avocet ' $S$ ' (Fig. 1). In the first trial involving two temperature treatments, the power model was used to present the variation observed in the response curves across entries and treatments. Temperature had a significant effect on stripe rust lesion development over time for Avocet 'S', MP152_4A, and AvS_4A. However, the stripe rust resistant cultivar Kariega produced similar lesion sizes at both incubation temperatures. The progression in lesion length responses observed in
MP152 4A, AvS 4A, and Avocet ' $S$ ' was similar at the lowtemperature incubation (Fig. 2A). However, analyses of data points 22 dpi showed that MP152_4A produced significantly shorter lesion lengths $(P<0.001)$ when compared with AvS_4A and Avocet ' $\mathrm{S}$ ' (Table 1). In the high-temperature treatment, the responses observed for both MP_152 and AvS_4A were similar and differed significantly $(P<0.001)$ from that of Avocet 'S', which was supported by analyses of the 22 dpi data points (Table 1; Fig. 2B). The host response of Kariega was a necrotic fleck at the point of inoculation
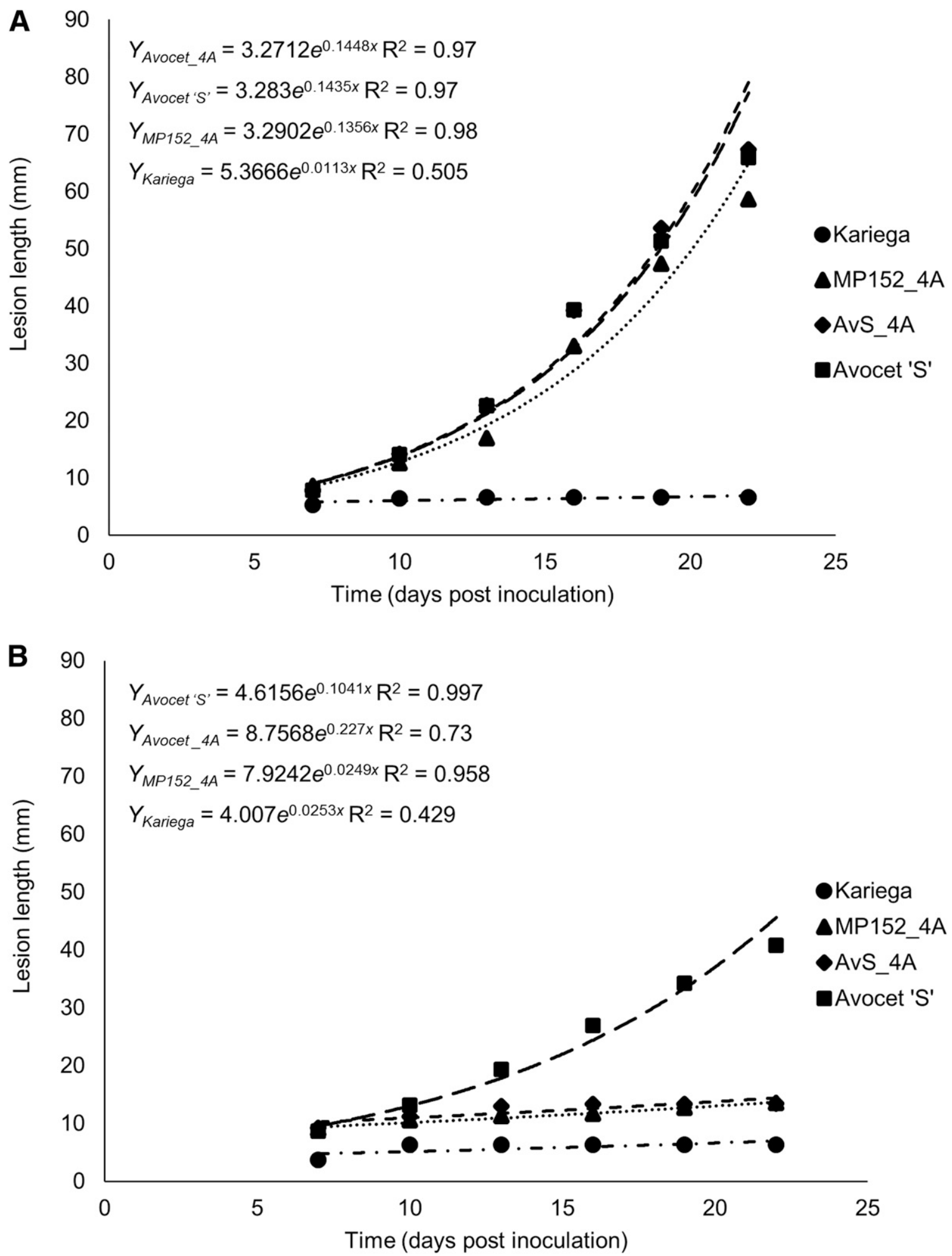

Fig. 2. Lesion length responses $(\mathrm{mm})$ of four wheat entries at mean low-temperature $\left(14.4\right.$ to $\left.17.8^{\circ} \mathrm{C}\right)(\mathbf{A})$ and mean high-temperature $\left(19.1\right.$ to $\left.23.7^{\circ} \mathrm{C}\right)(\mathbf{B})$ incubation treatments over a 22-day period post point inoculation with Puccinia striiformis f. sp. tritici race 6E22A+. 
independent of incubation temperature (Fig. 3). A slight increase in lesion length on Kariega flag leaves only occurred between 7 and $10 \mathrm{dpi}$ for both treatments, and the lesion length remained constant at 6.59 and $6.32 \mathrm{~mm}$ for the low and high incubation temperature treatments, respectively, from day 13 to day 22 post inoculation. Avocet ' $S$ ' produced compatible host infection types at both temperature regimes. However, lesion development on flag leaves of Avocet ' $S$ ' was delayed by the high-temperature regime $(40.82 \mathrm{~mm})$ when compared with the low-temperature regime $(65.93 \mathrm{~mm})$ but remained significantly longer in expansion when compared with that of the other entries, which did not exceed $13.6 \mathrm{~mm}$ in this environment. The lines MP_152 and AvS_4A produced compatible host responses at the low incubation temperature, but a necrotic response was observed at the high incubation temperature for both lines.

In the second trial, the logistic model best described the sigmoidal curve responses for Avocet 'S', Avocet 'R', Jupateco 'S', Krokodil, JIC871, and Jupateco 'R'. However, no sigmoidal response was found for the resistant cultivar Trident with lesion lengths that remained constant at $6.52 \mathrm{~mm}$ from 7 to $27 \mathrm{dpi}$. The curve responses observed in Avocet 'S', Avocet 'R', Jupateco 'S', Krokodil, and JIC871 clustered together, whereas the Jupateco 'R' response was independent of a group (Fig. 4). ANOVA for data points 27 dpi support these groupings. Trident $(6.52 \mathrm{~mm})$ produced significantly shorter lesion lengths $(P<0.001)$ followed by Jupateco ' $\mathrm{R}$ ' $(40.98 \mathrm{~mm})$. The third group included Avocet ' $\mathrm{R}$ ' $(80.24 \mathrm{~mm})$, Jupateco ' $\mathrm{S}$ ' (82.07 mm), Krokodil (85.12 mm), and Avocet ' $\mathrm{S}$ ' $(88.26 \mathrm{~mm})$, with JIC871 $(98.07 \mathrm{~mm})$ producing the longest lesion lengths. A box plot representing lesion length data of the respective entries 27 dpi further confirms the grouping of the entries (Fig. 5). Trident produced strong resistant phenotypes at the point of inoculation visible as chlorotic and necrotic flecks. The host response for Jupateco ' $\mathrm{R}$ ' was strongly necrotic, with the remaining entries producing compatible phenotypes. Comparative phenotypes for Trident, Jupateco 'R', Krokodil, Avocet 'S', and JIC871 27 dpi are shown in Figure 6.

\section{Discussion}

A flag leaf point inoculation technique, allowing for the reliable measuring and comparison of stripe rust lesion expansion under controlled conditions, was developed. One of the most noteworthy advantages of this technique is that flag leaves similar in age and appearance can be selected, which adds to accuracy and precision of phenotyping. Knowing the exact point of inoculation permits the reliable measurement of lesion expansion in wheat leaves where systemic colonization over time occurs. In this study, lesion length was easy to determine and was defined to leaf areas covered with sporulating pustules and clearly visible necrotic or surrounding chlorotic areas associated with infection. In wheats displaying a strong hypersensitive response without substantial expansion of a sporulating leaf area, final lesion size can be described, measured, and statistically compared. Disc application did not result in inoculum run-off away from the initial point of application, as was experienced in optimization trials with liquid suspensions (Boshoff, unpublished data). The paper discs are inexpensive, and their application is uncomplicated and quick. Furthermore, the technique does not require a dew chamber cycle, which often limits the number of pots, entries, and treatments manageable on a given day. The waterproof tape retained sufficient moisture in the discs over a 48-h incubation period, was easy to apply and remove, and did not show any signs of phytotoxicity.

The technique in its current form did not consider standardization of the exact spore load per disc. The number of spores that successfully infects the plant within the 6-mm disc area may vary. Measures to optimize or standardize spore load per disc could be considered in future studies to allow for comparison of entries differing in components of resistance such as initial infection rate. Necrosis observed at the point of inoculation for some of the infection points did not result in any significant differences in lesion expansion and can be attributed to plant cell death as lesions aged. Measuring lesion size at regular intervals on many flag leaves was tedious. However, depending on the objective of an experiment, the final lesion dimensions may be enough to discriminate among trial entries. Should more detailed comparisons be required over a time course (e.g., the characterization of a slow rusting response), more regular ratings will be necessary. Here, the application of automated phenotyping technology should be useful.

Previous point inoculation methods applied to achieve uniform infections with $P$. striiformis have been less successful when considering the success rate of infections achieved (Broers and Lopez-Atilano 1994; Milus et al. 2009). The use of agar blocks or agar solutions may also result in colonization and contamination by other microorganisms at the point of inoculation. The technique described by Sørensen et al. (2016) was efficient and resulted in a 100\% infection rate of $P$. striiformis on wheat seedlings. Resources required for fixing a large

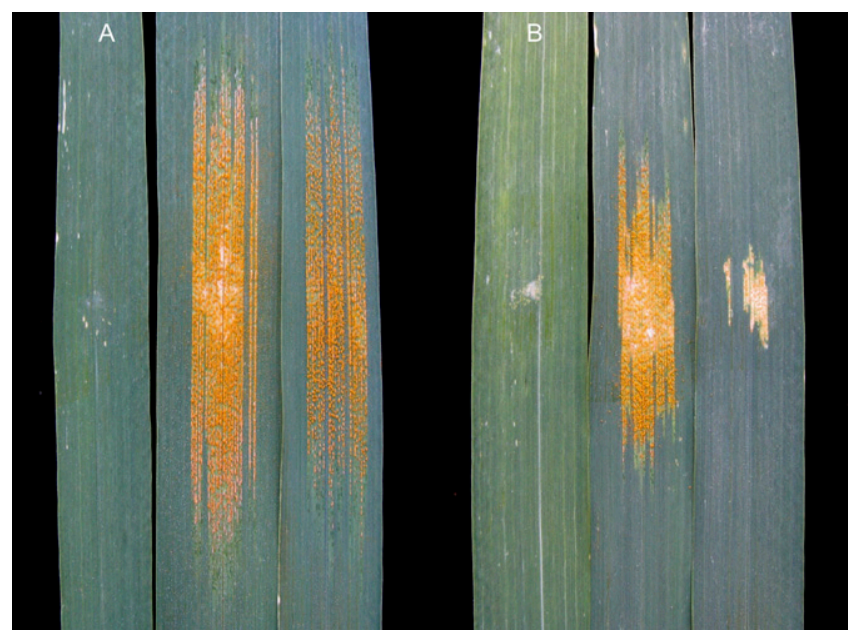

Fig. 3. Flag leaves from left to right showing representative stripe rust infection types and lesion lengths for the wheat entries Kariega, Avocet 'S', and AvS_4A 22 days post point inoculation with Puccinia striiformis f. sp. tritici race 6E22A+. The mean post inoculation night/day temperatures were at a mean low $\left(14.4\right.$ to $\left.17.8^{\circ} \mathrm{C}\right)(\mathbf{A})$ and mean high $\left(19.1\right.$ to $\left.23.7^{\circ} \mathrm{C}\right)(\mathrm{B})$, respectively.

Table 1. Two-way interaction in lesion length between four wheat entries incubated at low and high night/day temperature regime incubation treatments 22 days post point inoculation with Puccinia striiformis f. sp. tritici race $6 \mathrm{E} 22 \mathrm{~A}+{ }^{\mathrm{z}}$

\begin{tabular}{|c|c|c|c|}
\hline \multirow[b]{2}{*}{ Cultivar } & \multicolumn{2}{|c|}{ Temperature treatment $\left({ }^{\circ} \mathrm{C}\right)$} & \multirow[b]{2}{*}{ Cultivar mean } \\
\hline & 14.4 to $17.8^{\circ} \mathrm{C}$ night/day regime & 19.1 to $23.7^{\circ} \mathrm{C}$ night/day regime & \\
\hline Kariega & $6.59 \mathrm{a}$ & $6.32 \mathrm{a}$ & $6.46 \mathrm{a}$ \\
\hline MP152_4A & $58.64 \mathrm{~b}$ & $13.57 \mathrm{~b}$ & $36.11 \mathrm{~b}$ \\
\hline AvS_4A & $67.29 \mathrm{c}$ & $13.45 \mathrm{~b}$ & $40.37 \mathrm{c}$ \\
\hline Avocet 'S' & $65.93 \mathrm{c}$ & $40.82 \mathrm{c}$ & $53.37 \mathrm{~d}$ \\
\hline Treatment mean & $49.61 \mathrm{~b}$ & $18.54 \mathrm{a}$ & 34.08 \\
\hline
\end{tabular}

${ }^{\mathrm{z}}$ Mean values followed by the same letter in the respective columns as well as for the treatment means do not differ significantly according to Fisher's LSD $(P<$ $0.05)$. $\mathrm{LSD}=$ least significant difference at $0.05 \%$. Cultivar $\times$ treatment $=2.68$; cultivar mean (overall treatments $)=4.24$; and treatment mean (overall cultivars $)=$ 3.00 . 
number of leaves on a horizontal plate limit the number of entries that can be handled, and the technique is considered less practical when working with adult plants owing to variation in plant height and leaf position. Because the current point inoculation technique allows for the retention of moisture at the inoculation point, plants can be incubated under conditions of low humidity without affecting infection negatively. This facilitates the handling of a larger number of plants per inoculation cycle, because dew cabinets often are restricted in capacity.
The significant shorter mean stripe rust lesion development measured at the higher temperature regime for Avocet ' $S$ ' clearly shows the sensitivity of $P$. striiformis race 6E22A+ to higher ambient temperatures. Previous efforts to detect any resistance response in $\mathrm{AvS}$ 4A under greenhouse conditions failed. These experiments were carried out on adult plants using the spray inoculation method under a low-temperature regime ( 15 to $18^{\circ} \mathrm{C}$ night/day temperature regimes). The results obtained with the current point inoculation method at the low night/day temperature regime $\left(14.4\right.$ to $\left.17.8^{\circ} \mathrm{C}\right)$ confirmed

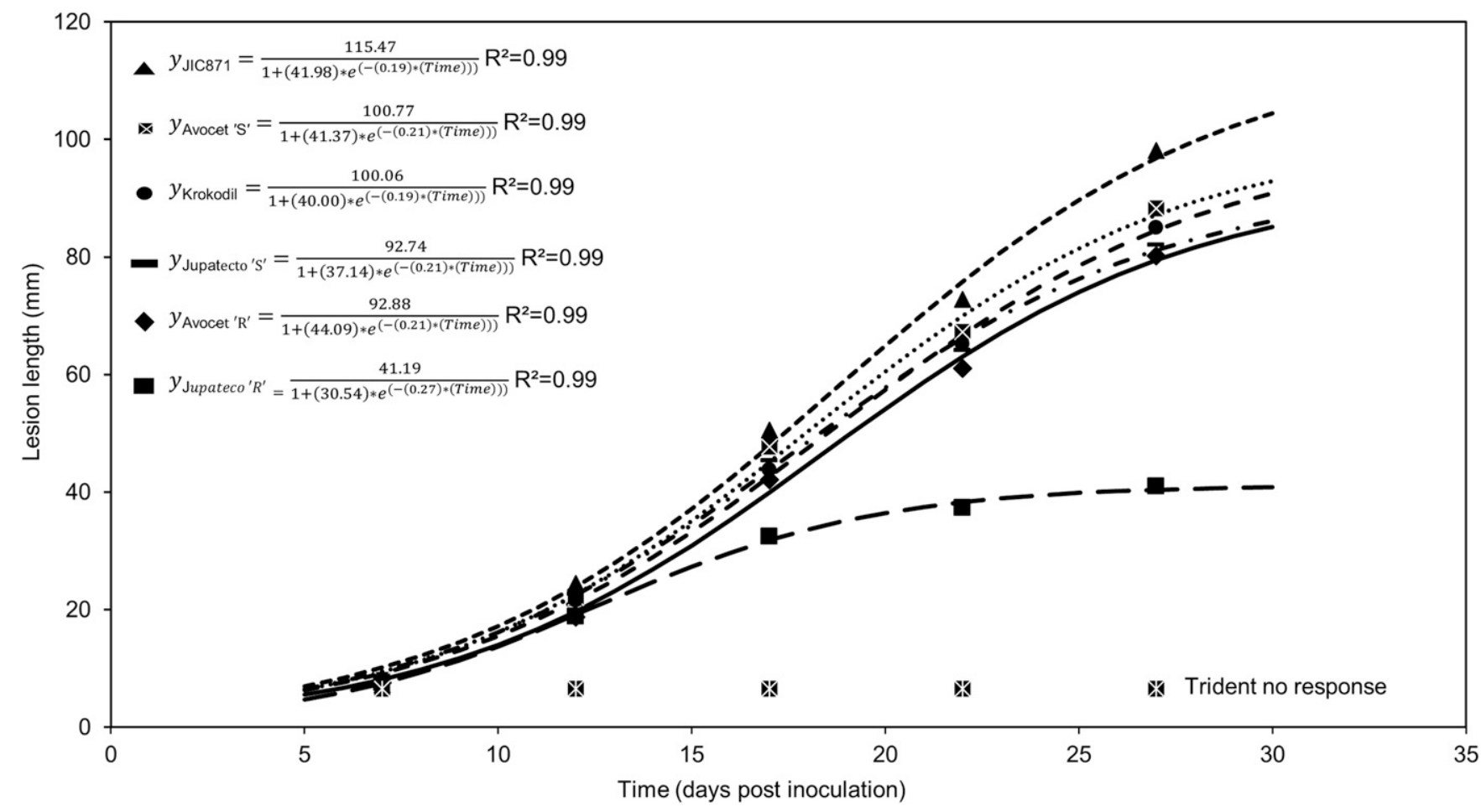

Fig. 4. Lesion length responses $(\mathrm{mm})$ of seven wheat entries over a 27-day period post point inoculation with Puccinia striiformis $\mathrm{f}$. sp. tritici race $6 \mathrm{E} 22 \mathrm{~A}+$.

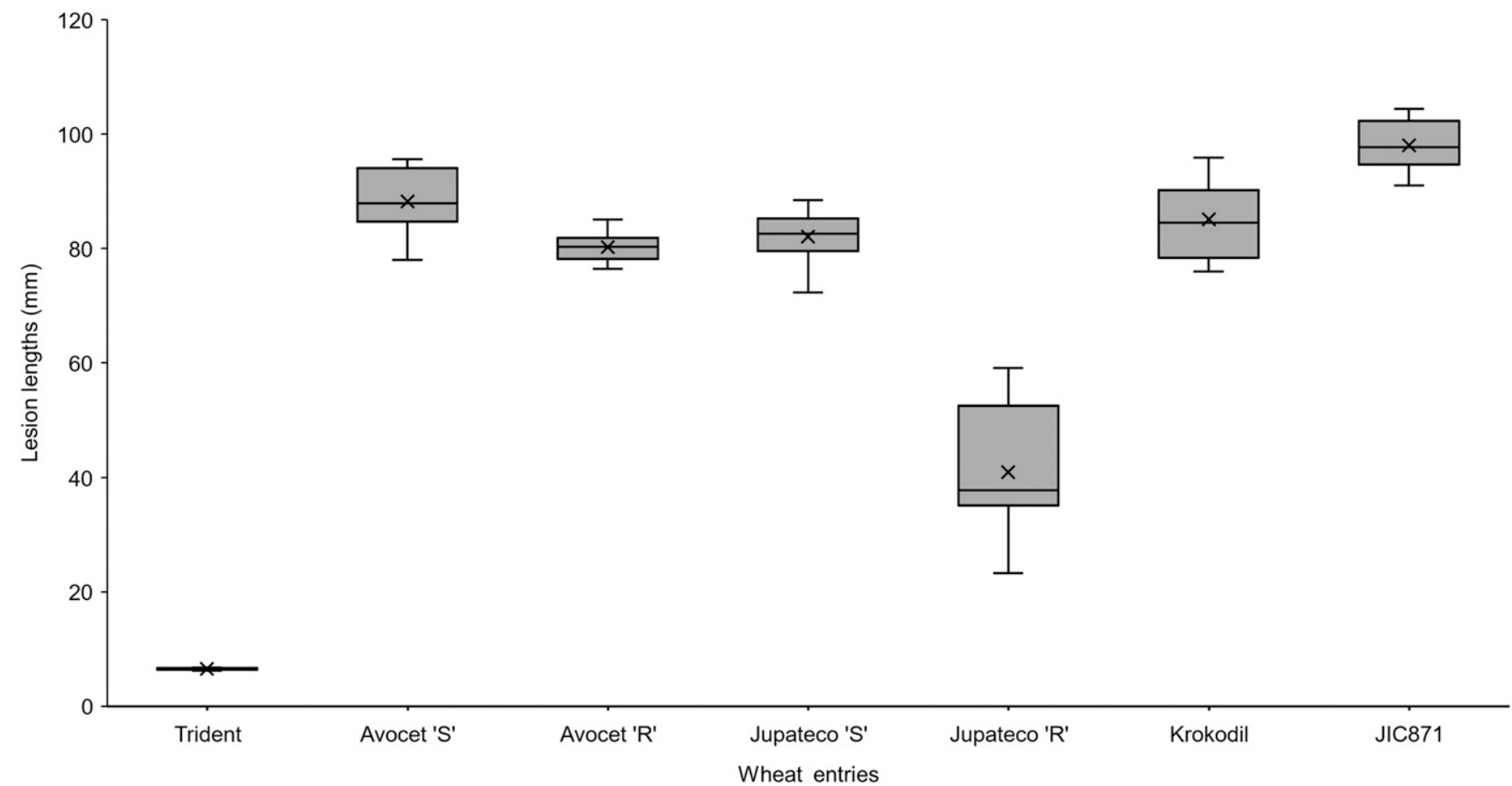

Fig. 5. Box plot representing lesion length of seven wheat entries 27 days post point inoculation with Puccinia striformis f. sp. tritici race $6 \mathrm{E} 22 \mathrm{~A}+$. Means are marked with $\mathrm{X}$, and error bars indicate data falling outside the upper and lower quartiles. 


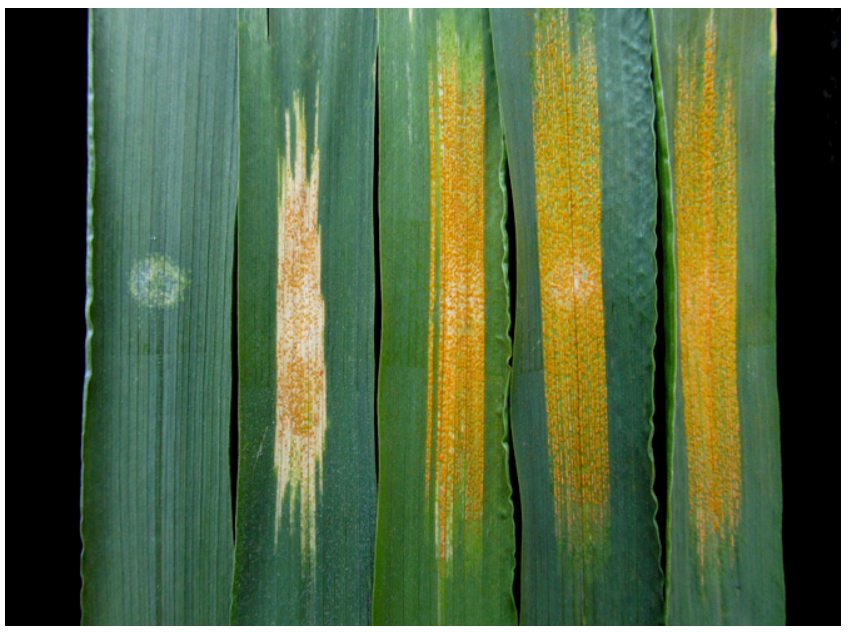

Fig. 6. Flag leaves from left to right showing representative stripe rust infection types and lesion lengths for the wheat entries Trident, Jupateco 'R', Krokodil, Avocet 'S', and JIC871 27 days post point inoculation with Puccinia striformis f. sp. tritici race 6E22A+. The mean post inoculation night/day temperatures maintained were 14.6 and $18.5^{\circ} \mathrm{C}$, respectively.

previous results (Pretorius, unpublished data) obtained with spray inoculation indicating that resistance conferred by the QYr.sgi-4A.1 QTL against stripe rust is not expressed at prevailing cooler temperatures. The necrotic response observed at the higher temperature regime $\left(19.1\right.$ to $\left.23.7^{\circ} \mathrm{C}\right)$ for MP152_4A and AvS_4A indicates that resistance conferred by this QTL is temperature sensitive. Follow-up trials to induce expression of the QYr.sgi-4A.1 resistance at an earlier growth stage in AvS_4A and MP152_4A by incubating inoculated seedlings at three temperature regimes ( 20 to 25,18 to 21 , and 15 to $18^{\circ} \mathrm{C}$, respectively) were unsuccessful (Boshoff, unpublished data). Resistance conferred by the QYr.sgi-4A.1 QTL therefore appears to be influenced by both growth stage and temperature, which is typical for high-temperature adult-plant stripe rust resistance reviewed by Chen (2013). This has implications for phenotypic expression of resistance under field conditions because temperature varies within and between seasons.

Results obtained with the point inoculation technique indicated the successful characterization of different stripe rust response types in adult wheat plants. Numerous applications for detailed phenotyping are possible: for example, routine germplasm screening including other parameters such as latent period and host reaction type, determining environmental influences, the effects of different $P$. striiformis races on selected germplasm, and even the possibility of field studies provided point inoculation occurs before natural infection. The technique further holds potential in surveillance of APR genes that are routinely ignored in standard race typing.

\section{Literature Cited}

Andres, M. W., and Wilcoxson, R. D. 1984. A device for uniform deposition of liquid suspended urediospores on seedling and adult cereal plants. Phytopathology 74:550-552.

Bender, C. M., Prins, R., and Pretorius, Z. A. 2016. Development of a greenhouse screening method for adult plant response in wheat to stem rust. Plant Dis. 100: 1627-1633.

Broers, L. H. M., and Lopez-Atilano, R. 1994. A method of inoculating adult wheat plants with urediospores of Puccinia striiformis to measure components of resistance. Plant Dis. 78:353-357.

Browder, L. E. 1971. Pathogenic specialization in cereal rust fungi, especially Puccinia recondita $\mathrm{f}$. sp. tritici, concepts, methods of study, and application. Agricultural Research Service Technical Bulletin No. 1432. U.S. Department of Agriculture, Washington, DC.

Chen, X. M. 2005. Epidemiology and control of stripe rust (Puccinia striiformis f. sp. tritici) on wheat. Can. J. Plant Pathol. 27:314-337.

Chen, X. M. 2013. High-temperature adult-plant resistance, key for sustainable control of stripe rust. Am. J. Plant Sci. 4:608-627.
Dong, Z., Hegarty, J. M., Zhang, J., Zhang, W., Chao, S., Chen, X., Zhou, Y., and Dubcovsky, J. 2017. Validation and characterization of a QTL for adult plan resistance to stripe rust on wheat chromosome arm 6BS ( $Y r 78)$. Theor. Appl. Genet. 130:2127-2137.

Ellis, J. G., Lagudah, E. S., Spielmeyer, W., and Dodds, P. N. 2014. The past, present and future of breeding rust resistant wheat. Front. Plant Sci. 5:641.

Eyal, Z., Clifford, B. C., and Caldwell, R. M. 1968. A settling tower for quantitative inoculation of leaf blades of mature small grain plants with urediospores. Phytopathology 58:712-714.

Hickey, L. H., Wilkinson, P. M., Knight, C. R., Godwin, I. D., Kravchuk, O. Y., Aitken, E. A. B., Bansal, U. K., Bariana, H. S., DeLacy, I. H., and Dieters, M. J. 2012. Rapid phenotyping for adult-plant resistance to stripe rust in wheat. Plant Breed. 131:54-61

Hintze, J. L. 2007. NCSS 2007. NSCC, Kaysville, UT. https://www.ncss.com/.

Klarquist, E. F., Chen, X. M., and Carter, A. H. 2016. Novel QTL for stripe rust resistance on chromosomes $4 \mathrm{~A}$ and $6 \mathrm{~B}$ in soft white winter wheat cultivars. Agronomy (Basel) 6:2-14.

Kloppers, F. J., and Pretorius, Z. A. 1997. Effects of combinations amongst genes Lr13, Lr34 and Lr37 on components of resistance in wheat to leaf rust. Plant Pathol. 46:737-750.

Michelmore, R. W., Christopoubou, M., and Caldwell, K. S. 2013. Impacts of resistance gene genetics, function, and evolution on a durable culture. Annu. Rev. Phytopathol. 51:291-319.

Milus, E. A., Kristensen, K., and Hovmøller, M. S. 2009. Evidence for increased aggressiveness in a recent widespread strain of Puccinia striiformis $\mathrm{f}$. sp. tritici causing stripe rust of wheat. Phytopathology 99:89-94.

Mortensen, K., Green, G. J., and Atkinson, J. 1979. A method for uniform infection of seedlings and adult cereal plants by Puccinia graminis $\mathrm{f}$. $\mathrm{sp}$ tritici. Phytopathology 69:420-423.

Mundt, C. C. 2018. Pyramiding for resistance durability: Theory and practice. Phytopathology 108:792-802.

Pretorius, Z. A., Ayliffe, M., Bowden, R. L., Boyd, L. A., DePauw, R. M., Jin, Y., Knox, R. E., McIntosh, R. A., Park, R. F., Prins, R., and Lagudah, E. S. 2017. Advances in control of wheat rusts. Pages 295-343 in: Achieving Sustainable Cultivation of Wheat, Vol. 1: Breeding, Quality Traits, Pests and Diseases. P. Langridge, ed. Burleigh Dodds Science Publishing, Cambridge, U.K.

Pretorius, Z. A., Park, R. F., and Wellings, C. R. 2000. An accelerated method for evaluating adult-plant resistance to leaf and stripe rust in spring wheat. Acta Phytopathol. Entomol. Hung. 35:359-364.

Pretorius, Z. A., Pienaar, L., and Prins, R. 2007. Greenhouse and field assessment of adult plant resistance in wheat to Puccinia striiformis f. sp. tritici. Australas. Plant Pathol. 36:552-559.

Prins, R., Dreisigacker, S., Pretorius, Z. A., van Schalkwyk, H., Wessels, E., Smit, C., Bender, C., Singh, D., and Boyd, L. A. 2016. Stem rust resistance in geographically diverse collection of spring wheat lines collected from across Africa. Front. Plant Sci. 7:973.

Riaz, A., Periyannan, S., Aitken, E., and Hickey, L. 2016. A rapid phenotyping method for adult plant resistance to leaf rust in wheat. Plant Methods 12:17.

Singh, D., McIntosh, R. A., and Park, R. F. 1996. An adhesive tape technique for inoculating adult-plants of wheat with rust pathogens. Pages 150-152 in: Proceedings of the $8^{\text {th }}$ Assembly of Wheat Breeding Society. R. A Richards, C. W. Wrigley, H. M. Rawson, G. J. Rebetzke, J. L. Davidson, and R. S. Brettell, eds. Wheat Breeding Society of Australia, Canberra, Australia.

Singh, R. P. 1992. Genetic association of leaf rust resistance gene $L r 34$ with adult plant resistance to stripe rust in bread wheat. Phytopathology 82:835-838.

Singh, R. P., Herrera-Foessel, S., Huerta-Espino, J., Singh, S., Bhavani, S., Lan, C., and Basnet, B. R. 2014. Progress towards genetics and breeding for minor genes based resistance to Ug99 and other rusts in CIMMYT highyielding spring wheat. J. Integr. Agric. 13:255-261.

Singh, R. P., Hodson, D. P., Jin, Y., Lagudah, E. S., Ayliffe, M. A., and Bhavani, S. 2015. Emergence and spread of new races of wheat stem rust fungus: Continued threat to food security and prospects of genetic control. Phytopathology 105: 872-884.

Smit, H. A., Tolmay, V. L., Barnard, A., Jordaan, J. P., Koekemoer, F. P., Otto, W. M., Pretorius, Z. A., Purchase, J. L., and Tolmay, J. P. C. 2010. An overview of the context and scope of wheat (Triticum aestivum) research in South Africa from 1983 to 2008. S. Afr. J. Plant Soil 27:81-96.

Sørensen, C. K., Thach, T., and Hovmøller, M. S. 2016. Evaluation of spray and point inoculation methods for the phenotyping of Puccinia striiformis on wheat. Plant Dis. 100:1064-1070.

Visser, B., Herselman, L., and Pretorius, Z. A. 2016. Microsatellite characterisation of South African Puccinia striiformis races. S. Afr. J. Plant Soil 33:161-166.

Wellings, C. R., McIntosh, R. A., and Hussain, M. 1988. A new source of resistance to Puccinia striiformis f. sp. tritici in spring wheats (Triticum aestivum). Plant Breed. 100:88-96.

Zadoks, J. C. 1961. Yellow rust on wheat: Studies in epidemiology and physiologic specialization. Tijdschr. Planteziekten 67:69-258. 